\title{
Travaux inédits
}

Travaux soutenus devant les universités portant sur la période révolutionnaire (vers 1750-vers 1830)

\section{Pascal Dupuy}

\section{(2) OpenEdition \\ 1 Journals}

Édition électronique

URL : https://journals.openedition.org/ahrf/11031

DOI : 10.4000/ahrf.11031

ISSN : 1952-403X

Éditeur :

Armand Colin, Société des études robespierristes

Édition imprimée

Date de publication : 1 décembre 2008

Pagination : 177-196

ISSN : 0003-4436

\section{Référence électronique}

Pascal Dupuy, «Travaux inédits », Annales historiques de la Révolution française [En ligne], 354 | octobredécembre 2008, mis en ligne le 14 décembre 2009, consulté le 24 avril 2022. URL : http:// journals.openedition.org/ahrf/11031 ; DOI : https://doi.org/10.4000/ahrf.11031 


\title{
TRAVAUX INÉDITS
}

\section{TRAVAUX SOUTENUS DEVANT LES UNIVERSITÉS PORTANT SUR LA PÉRIODE RÉVOLUTIONNAIRE (VERS 1750-VERS 1830) ${ }^{1}$}

Pascal DupuY

\author{
AMIENS \\ Université de Picardie-Jules Verne \\ 2006
}

Denis Emmanuelle, Le tribunal du district de Montdidier pendant la Révolution française (1790-1795), master 1, ss dir. G. Hurpin et S. Beauvalet, 2006.

Devisme Samuel, Le tribunal de district d'Amiens pendant la Révolution française (1790-1795), master 1, ss dir. G. Hurpin et S. Beauvalet, 2006.

\section{7}

Denis Emmanuel, L'activité du tribunal civil du district de Montdidier, 17911795, master 2, ss dir. S. Beauvalet, 2007.

Devisme Samuel, L'activité du tribunal civil du district d'Amiens, 1791-1795, master 2, ss dir. S. Beauvalet, 2007.

\section{ANGERS}

UNIVERSITÉ D'ANGERS

2007

Banchereau Pierrick, Le consulat de France à Alger de 1780 à 1789, master 1, ss dir. F. Brizay et P. Haudrère, 2007.

(1) Les $A H R F$ remercient pour l'envoi des listes de travaux soutenus dans leur université : Marc Bélissa, Michel Biard, Frédéric Bidouze, Philippe Bourdin, Serge Brunet, Paul Chopelin, Natacha Coquery, Benoit Marpeau, Guy Martinière, Anne de Mathan, Matthieu de Oliveira, François Pernot, Jean-Paul Rothiot, Véronique Sarrazin, Éric Saunier, Pierre Serna. 
Bellanger Florian, Les relations commerciales avec Tunis (1750-1800), master 1, ss dir. F. Brizay et V. Sarrazin, 2007.

Briand-Royer Pauline, Le consulat de France à Alger entre 1730 et 1780, master 1, ss dir. F. Brizay et P. Haudrère, 2007.

Elbisser Sarah, La politique internationale dans la presse angevine pendant la Révolution, master 1, ss dir. V. Sarrazin et F. Brizay, 2007.

Ferre Charline, La pratique testamentaire à Vernoil le Fourrier de 1671 à 1789, master 1, ss dir. P. Haudrère, 2007.

Lachere Marie-Céline, La vie quotidienne à Saint-Domingue et l'esclavage d'après la correspondance de Jacques Hilaire Margariteau, Angevin, conseiller au Conseil supérieur de Saint-Domingue, 1769-1786, master 1, ss dir. P. Haudrère, 2007.

Seyeux Gaëlle, Les mentalités religieuses à traves les testaments des Ponts-de-Cé de 1770 à 1790, master 1, ss dir. P. Haudrère, 2007.

\section{8}

Barbaud Angélique, Le commerce français à Tunis entre 1789 et 1792, master 1, ss dir. F. Brizay et V. Sarrazin, 2008.

Blusseau Marie, Les spectacles publics, les divertissements et loisirs collectifs à Angers dans la seconde moitié du XVIII siècle, master 1, ss dir. V. Sarrazin et F. Brizay, 2008.

Boistel Romain, Les relations entre Raguse et la France de 1772 à 1810, master 1, ss dir. F. Brizay et V. Sarrazin, 2008.

Briand-Royer Pauline, Le consulat de France à Alger entre 1689 et 1776, master 2, ss dir. F. Brizay et P. Haudrère, 2008.

Doucet Mathieu, Saumur, une bibliothèque municipale de province dans la première moitié du XIX $X^{\mathrm{e}}$ siècle, master 1, ss dir. P. Quincy-Lefebvre et V. Sarrazin, 2008.

Duffay Amélie, Les pratiques testamentaires entre Loir et Sarthe d'après les minutes des notaires de Tiercé de 1730 à 1790, master 1, ss dir. P. Haudrère, 2008.

Guilleret Jordan, Le marquis de Villebois, Angevin et gouverneur de la Guyane française, 1732-1788, master 1, ss dir. P. Haudrère, 2008.

Peycere Lucile, La politique dans les Affiches d'Angers sous l'Ancien Régime, 1773-1787, master 1, ss dir. V. Sarrazin et P. Quincy-Lefebvre, 2008.

Rondeau Valentin, Le commerce français en Méditerranée à l'épreuve de la course barbaresque dans la seconde moitié du XVIII siècle, master 1, ss dir. F. Brizay et V. Sarrazin, 2008. 


\section{ARRAS \\ UNIVERSITÉ D'ARTOIS \\ 2006}

Lallain Clément, L'administration municipale dans le Pas-de-Calais sous le Directoire (an IV-an VIII), master 2, ss dir. A. Crépin, 2006.

Mathieu Céline, Clergé et paroisses du Pas-de-Calais en 1790 - District de Béthune. Essai de statistiques et de cartographie, master 1, ss dir. G. Deregnaucourt, 2006.

\section{7}

Déruguillier Fany, La vie matérielle de la noblesse dans le Béthunois au XVIII siècle : l'exemple de quatre familles d'après les inventaires révolutionnaires, master 1, ss dir. G. Deregnaucourt et L. Baudoux, 2007.

\section{BOULOGNE-SUR-MER \\ Université du LitToral Côte d'Opale 2006}

Holuigue Fanny, Les corsaires pendant la Révolution 1794-1797, master 1, ss dir. P. Villiers, 2006.

\section{7}

François Pittini, Le regard de Napoléon sur la marine et la mer, master 2, ss dir. P. Villiers, 2007

\section{BREST}

\section{Université de Bretagne OCCIDENTALE}

\section{5}

Brulé Héloïse, Tuteurs et enfants orphelins dans la sénéchaussée de Lesneven, 1785-1787, master 1, ss dir. P. Jarnoux, A. de Mathan, 2005.

Coquil Nadège, Des étrangers au bagne de Brest : étude des arrivées étrangères entre l'an XI et 1814, master 1, ss dir. P. Jarnoux, A. de Mathan, 2005.

Couliou Hélène, Les Girondins au tribunal de l'histoire. Historiographie des Girondins aux XIX et $X X^{\mathrm{e}}$ siècles: confirmation ou révision du procès de 1793 ?, master 1, ss dir. A. de Mathan et Y. Tranvouez, 2005.

Gosselin-Lullien Marie-Christine, La marine, les forestiers, les populations: leurs devoirs et leur quotidien dans les forêts royales de la maîtrise de Carhaix, 1680-1750, master 1, ss dir. P. Jarnoux, 2005.

Kéruzec Valérie, La postérité des partisans des Girondins dans le Finistère, master 1, ss dir. A. de Mathan et J.-Y. Carluer, 2005. 
Le Fourn Delphine, La tutelle des mineurs dans les juridictions royales des sénéchaussées de Châteauneuf-du-Faou et Châteaulin de 1780 à 1783, master 1, ss dir. P. Jarnoux, A. de Mathan, 2005.

Le Quéré Rozenn, Société rurale et événement maritime à Plozévet. Monographie d'une famille de domaniers (1700-1789), Master 1, ss dir. F. Roudaut, 2005.

Vaillant Morgane, Étude du travail féminin à l'arsenal de Brest au XVIII siècle, 1753-1764, master 1, ss dir. P. Jarnoux, 2005.

\section{6}

Bantas Cécile, Le marché immobilier brestois à la fin de l'Ancien Régime, master 1, ss dir. P. Jarnoux, P. Pourchasse, 2006.

Bertholoux Jenny, Assassins et meurtriers au bagne de Brest entre 1770 et 1799 selon les registres matricules du bagne, master 1, ss dir. P. Jarnoux, A. de Mathan, 2006.

El Kholi Hugo, Comprendre l'historiographie du libéralisme politique moderne : l'école de Cambridge en débat, master 1, ss dir. F. Bouthillon, A. de Mathan 2006.

Herry Aurélie, La Russie à l'est de l'Oural au XVIII' siècle vue par les voyageurs (2 vol.), master 1, ss dir. P. Pourchasse, P. Jarnoux, 2006.

Riou Yann, La politique navale de Maurepas : l'exemple de l'arsenal de Brest (1737-1744), master 1, ss dir. P. Pourchasse, P. Jarnoux, 2006.

Seveno Aude, La Laponie et les Lapons vus par les voyageurs du XVIT et XVIIT siècles (2 vol.), master 1, ss dir. P. Pourchasse, P. Jarnoux, 2006

\section{7}

Bras Nolwenn, L'armement pour la pêche à Terre-Neuve dans les ports de la baie de Saint-Brieuc entre 1763 et 1789, master 1, ss dir. P. Pourchasse, P. Jarnoux, 2007.

Lannuzel Gaëtan, La Marine et l'arsenal de Brest au temps de la Régence (17151723) master 1, ss dir. P. Pourchasse, P. Jarnoux, 2007.

Le Gall Marjorie, Regards français sur l'avènement de Catherine II, impératrice de Russie, 1762-1774, master 1, ss dir. P. Pourchasse, P. Jarnoux, 2007.

Riou Gwendal, La politique navale de Rouillé : l'exemple de l'arsenal de Brest (1749-1754), master 1, ss dir. P. Jarnoux, P. Pourchasse, 2007.

\section{8}

Abasq Romain, Les étrangers dans le Finistère, 1793-an II, master 1, ss dir. A. de Mathan, P. Pourchasse, 2008

Baron Bruno, Les élites municipales brestoises, 1750-1820, master 2, ss dir. P. Jarnoux, A. de Mathan, 2008 
Bikfalvi Marianne, François Deshaies de Montigny : officier et diplomate français aux Indes (1776-1792), master 1, ss dir. P. Pourchasse, A. de Mathan, 2008.

Cadio Émilie, Les députés du Finistère à la Convention nationale, master 1, ss dir. A. de Mathan, P. Jarnoux, 2008

Celton Aurélie, Idéaux et réalités de la justice politique sous la Terreur, l'exemple du tribunal révolutionnaire de Brest, master 1, ss dir. A. de Mathan, P. Jarnoux, 2008.

Combot Gaël, Correspondance de Léonard Bousogne, commerçant brestois sous le Directoire et le Consulat (2 vol.), master 1, ss dir. P. Pourchasse, A. de Mathan, 2008.

Demez Florian, L'intérêt stratégique des Îles Mascareignes et plus précisément de l'île de France pendant le Premier Empire d'après les rapports de son capitaine général, Charles Mathieu Isidore Decaen, master 1, ss dir. P. Pourchasse, P. Jarnoux, 2008.

Kerdreux Tanguy, Les naufrages des navires de l'Europe du Nord sur les rivages de l'Amirauté de Cornouaille au XVIII siècle, master ss dir. P. Pourchasse, P. Jarnoux, 2008.

Moal Gauthier, Les signaux. Le difficile problème de la communication lors des combats navals, master 1, ss dir. P. Pourchasse, P. Jarnoux, 2008.

Petton Linda, La pêche à la sardine à Concarneau au temps de la Révolution et de l'Empire, master 1, ss dir. P. Pourchasse, P. Jarnoux, 2008.

Tanguy Charlotte, Les croyances religieuses à Tahiti de l'arrivée des premiers Européens à la mort de la reine Pomaré $I V$, master 1, ss dir. P. Pourchasse, P. Jarnoux, 2008.

Turnbull Rhiannon, Le blocus britannique et la France révolutionnaire, master 1, ss dir. P. Pourchasse, P. Jarnoux, 2008.

Weber Audrey, La Basse-Louisiane (1757-1769), master 1, ss dir. P. Pourchasse, P. Jarnoux, 2008.

\section{CAEN \\ Université de CAen \\ 2008}

Ikhlef Benoît, La vie politique et l'opinion publique dans le Calvados au temps de la monarchie censitaire. De la première Restauration à la fin de la monarchie de Juillet (1814-1848), master 1, ss dir. J. Quellien et M. Guérin, 2008. 


\section{CERGY-PONTOISE \\ Université de Cergy-Pontoise \\ 2002}

Ravaud Ysabelle, Le clergé du grand vicariat de Pontoise de 1700 à 1750, mâ̂trise d'histoire moderne, ss dir. F. Pernot, 2002.

\section{3}

Ollivry Éric, L'idée d'Europe chez les physiocrates, maîtrise d'histoire moderne, ss dir. F. Pernot, 2003.

Rodier Simon, Le traité de Paris (1763) : une honte et une catastrophe pour la France? maîtrise d'histoire moderne, ss dir. F. Pernot, 2003.

\section{4}

Lecarpentier Jérémy, La réorganisation de la justice dans le district de Pontoise pendant la Révolution : entre permanences et mutations (1780-1799), mâ̂trise d'histoire moderne, ss dir. F. Pernot, 2004.

\section{5}

Bretonnière Jean-Baptiste, Projets de paix universelle en Europe et idée d'Europe chez les libres-penseurs français (La Motte Le Voyer, Cyrano de Bergerac, Pierre Bayle et Fontenelle) (1657-1757), maîtrise d'histoire moderne, ss dir. F. Pernot, 2005.

Briançon Sonia, La délinquance dans le bailliage de la Roche-Guyon de 1760 à 1791, maîtrise d'histoire moderne, ss dir. F. Pernot, 2005.

Ferreira Romain, La prison départementale de Pontoise au XIX $X^{\mathrm{e}}$ siècle, maîtrise d'histoire moderne, ss dir. F. Pernot, 2005.

Kevorkian Alexandre, Les projets de paix universelle et l'idée d'Europe à la fin du XVIII siècle, d'après Gargaz, Pallier de Saint-Germain et Anacharsis Cloots, maîtrise d'histoire moderne, ss dir. F. Pernot, 2005.

Le Guéné Gaëlle, Insécurité et délinquance dans le bailliage de Pontoise dans les vingt dernières années de l'Ancien Régime (de la Guerre des Farines à la Grande Peur), maîtrise d'histoire moderne, ss dir. F. Pernot, 2005.

Viton Juliette, La vie quotidienne dans le Vexin au XIX' siècle à partir des inventaires après décès (série B), maîtrise d'histoire moderne, ss dir. F. Pernot, 2005.

\section{6}

Antomori Élise, L'habitat rural dans le bailliage de Pontoise sous le règne de Louis XV, master 1, ss dir. F. Pernot, 2006.

Debras Claire, Les cours criminelles spéciales, master 1, ss dir. F. Pernot, 2006.

Koch Sonja, Pauvreté et charité dans la seigneurie de la Roche-Guyon (17671797), master 1, ss dir. F. Pernot, 2006. 
Leboucher Sophie, L'héritage de Pierre Du Bois, Sully, Comenius, William Penn, l'abbé de Saint Pierre, dans l'idée de confédération d'États (jusqu'au fédéralisme) du projet d'Europe de Coudenhove-Kalergi, master 1, ss dir. F. Pernot, 2006.

Rault Mélanie, Propriété et exploitation rurale à Boissy l'Aillerie 1750-1789, master 1, ss dir. F. Pernot, 2006.

Rebuffé Erwan, La correspondance de l'intendant Dumazy à l'époque de la duchesse D'Enville (1788-1792), master 1, ss dir. F. Pernot, 2006.

Zanon Fanny, Inventaire d'archives - Le bailliage royal de Beaumont-sur-Oise (1594-1789), master 1, ss dir. F. Pernot, 2006.

\section{CLERMONT-FERRAND \\ Université Blaise Pascal (Clermont-Ferrand II) \\ 2008}

Boudet Jonathan, Religion, pouvoirs et société dans la France napoléonienne : l'exemple du département du Puy-de-Dôme (1799-1814), master 1, ss dir. P. Bourdin, 2008.

Favier Jean-Baptiste, La haute administration du département de l'Allier entre le Consulat et la Restauration (an VIII-1830), master 2, ss dir. J.-P. Luis, 2008.

Pivoteau Sébastien, Châteaux et châtelains à la fin du XVIII siècle : l'exemple cantalien, master 2, ss dir. P. Bourdin, 2008.

Scafone Fabien, Les fêtes dans la Creuse pendant la Révolution et le Consulat, master 2, ss dir. P. Bourdin, 2008.

Taillecours Florian, Du champ de bataille au foyer. Les anciens soldats et les pensionnés militaires dans le Puy-de-Dôme sous la Révolution (17921799), master 1, ss dir. P. Bourdin, 2008.

\section{LA ROCHELLE, UNIVERSITÉ de LA Rochelle}

Ronsseray Céline, Administrer Cayenne: sociabilités, fidélités et pouvoirs des fonctionnaires coloniaux en Guyane française au XVIII siècle, thèse NR, ss dir. G. Martinière, 2007, 3 vols, 691 p.

\section{LE HAVRE \\ UNIVERSITÉ DU HAVRE}

2006

Baudry Cynthia, La vie sociale à Montivilliers au XVIII siècle, master 1, ss dir. É. Saunier et É. Wauters, 2006.

Fauvelle Micheline, Les marchands de bois de chauffage de la maîtrise particulière de Rouen au XVIIr siècle, master 1, ss dir. É. Wauters et É. Saunier, 2006. 
Venem Aurélie, Violence et criminalité au Havre au XVIII siècle, master 1, ss dir. É. Wauters et É. Saunier, 2006.

Dutot Marie-Jeanne, Les pratiques religieuses au Havre sous le Premier Empire, master 1, ss dir. É. Saunier et É. Wauters, 2006.

Longuemare Frédéric, Souvenirs d'un vieillard. Édition commentée du manuscrit de Pierre-Philippe-Urbain Thomas (1776-1845), master 1, ss dir. É. Saunier et É. Wauters, 2006.

Clatot Florent, Souvenirs d'un vieillard. Édition commentée du manuscrit de Pierre-Philippe-Urbain Thomas (1776-1845), master 1, ss dir. É. Saunier et É. Wauters, 2006.

Fontaine Tania, Violence et criminalité dans les sociétés portuaires haut-normandes sous l'Empire, master 2, ss dir. É. Saunier et É. Wauters, 2006.

\section{7}

Renault Agnès, La communauté française de Santiago de Cuba (1791-1825), thèse NR, ss dir. E. Wauters et A. Yacou, 2007, 2 vol., 888 p.

Rioult Florence, Les missionnaires français en Afrique du nord (1620-1789), master 2, ss dir. É. Wauters, 2007.

Tufel-Chopin Élise, La vie comme un roman. Témoignages et récits de vie à la fin $d u$ XVIII siècle, master 2, ss dir. É. Wauters, É. Saunier et R. de Filippis, 2007.

\section{LILLE \\ Université Charles-De-Gaulle (Lille III) \\ 2006}

Bodart Benoît, La justice militaire sous le Consulat et l'Empire, l'exemple de la première et de la seizième divisions militaires, master 1, ss dir. J.-F. Chanet, 2006

Choquet Thomas, Le problème de la reconversion des seigneurs laïcs par-delà la Révolution, master 1, ss dir. J.-P. Jessenne, 2006.

Côme Antoine, Paysans et villageois du Nord face aux événements et aux politiques révolutionnaires, master 1, ss dir. J.-P. Jessenne, 2006.

Dubois François, Le pouvoir municipal à Gravelines sous la Révolution (17891792), master 1, ss dir. P. Guignet, 2006.

Fiévet Isabelle, Des faux pour échapper à la conscription. Le tribunal criminel spécial du Nord et la répression des faux en matière de conscription (an $X$-1811), master 1, ss dir. H. Leuwers, 2006.

Kasdi Mohamed, La naissance de l'industrie cotonnière dans le département $d u$ Nord (1700-1830) : un produit, des consommateurs, des entrepreneurs, thèse NR, ss dir. G. Gayot, 2006.

Leduc Claire, Le divorce à Douai pendant la Révolution française (1792-1796) d'après les tribunaux de famille, master 1, ss dir. R. Grevet, 2006. 
Merveille Armand, L'assassinat de Gustave III, ses conséquences et son écho dans la France révolutionnaire, master 2, ss dir. R. Grevet, 2006.

Mesurolle Caroline, Les professions maritimes à Dunkerque en 1790, master 1, ss dir. D. Roselle, 2006.

Philippo Hervé, Fortunes, trajectoires et modes de vie des notables lillois (17801830), thèse NR ss dir. G. Gayot, 2006.

Pulpiton Virginie, L'Église réfractaire dans le canton de Valenciennes pendant la Révolution française, master 1, ss dir. R. Grevet, 2006.

Susset Géraldine, Pierre et Étienne Lejosne de l'Espierre : la Révolution, une affaire de famille (1775-1794) ?, master 1, ss dir. H. Leuwers, 2006.

Turek Anne-Sophie, Les évêques d'Ypres et de Tournai pendant la Révolution française (1789-1793), master 1, ss dir. H. Leuwers, 2006.

\section{7}

Benoît Robert, Les administrateurs du département $d u$ Nord, master 1, ss dir. H. Leuwers, 2007.

Brassart Laurent, La République à l'épreuve de l'ordinaire et de l'exception. État-Nation, pouvoirs locaux et mouvements collectifs dans le département de l'Aisne de 1791 à 1795, thèse NR, ss dir. J.-P. Jessenne, 2007

Cassan Audrey, Archives et archivistes à Lille du XVIIr siècle à la Restauration, master 1, ss dir. P. Guignet, 2007.

Condette Jean-François, Le recteur d'Académie, l'École, la Nation (1808-1940). Entre réalités scolaires locales et politiques éducatives nationales, Habilitation à diriger des recherches, 2007.

Deaucourt Marie et Rency Anne, Les retentissements de la fuite du roi Louis XVI sur les populations du Nord/Pas-de-Calais, master 1, ss dir. J.-P. Jessenne, 2007.

Dubois François, Le pouvoir municipal à Gravelines sous la Révolution (17921794), master 2, ss dir.P. Guignet, 2007

Guerre Karine, Les lois sociales de Ventôse an II dans le Nord, master 2, ss dir. G. Gayot et J.-P. Jessenne, 2007.

Kaci Maxime, L'entrée en politique, mots d'ordre et engagements entre Paris et le Nord en 1792, master 2, ss dir. J.-P. Jessenne, 2007.

Kowalski Sandra, La dénonciation dans le département du Nord pendant la Révolution française, (1793-an II), master 2, ss dir. R. Grevet, 2007.

Legrand Maximilien, Crimes et délits à Douai durant la Révolution française au travers des archives du tribunal de district (1791-1792), master 1, ss dir. H. Leuwers, 2007.

Lepers Justine, La rumeur en Révolution dans l'espace septentrional, master 2, ss dir. H. Leuwers, 2007. 
Leyssens Aldéric, La démographie à Tournai entre 1789 et 1799, master 1, ss dir. P. Guignet, 2007.

Siour Dorothée, Revendications politiques et sociales à l'apogée de la Terreur : septembre, décembre, master 1, ss dir. J.-P. Jessenne, 2007.

\section{LYON \\ Université Jean Moulin (Lyon III) \\ 2006}

Boccard Edwige, Les mission moraves en France de 1731 à 1794, d'après les correspondances d'Antoine Court, de Paul Rabaut et le Journal de l'Unité des Frères, master 1, ss dir. Y. Krumenacker, 2006.

Charignon Marie-Ange, Étude sur les récits de conversion entre catholiques et protestants en France durant l'époque moderne (1580-1795), master 1, ss dir. Y. Krumenacker, 2006.

Pattalocchi Alexandra, Crimes et délits sexuels portés en justice dans le Lyonnais (1750-1789), master 1, ss dir. Y. Krumenacker, 2006.

Pouradier-Duteil Pauline, La vie liturgique du chapitre Saint-Jean à Lyon aux $X V I I^{e}-X V I I I^{e}$ siècles, master 1, ss dir. B. Hours, 2006.

\section{7}

Bonnaud Aurélie, Les ursulines et les carmélites de Trévoux à l'époque moderne (XVII ${ }^{e}$-XVIII ${ }^{e}$ siècles), master 1, ss dir. B. Hours, 2007.

Bouvet Géraldine, La criminalité dans une juridiction péri-urbaine au XVIII siècle : l'exemple du chapitre Saint-Just à Lyon, master 1, ss dir. B. Hours, 2007.

Chenevier Christophe, Les négociants protestants lyonnais aux XVII et XVIII siècles, master 1, ss dir. Y. Krumenacker, 2007.

Couriol Étienne, Parenté spirituelle et réseaux sociaux à Lyon aux XVII et XVIII siècles, master 2, ss dir. B. Hours, 2007.

Gallet de Santerre Stanislas, Le régiment de Santerre au XVII et au XVIII siècle, master 1, ss dir. P. J. Souriac, 2007.

Guillet-Lhomat Pierre, Le district de Lyon-Campagne. Son rapport avec les institutions après le siège de Lyon (octobre 1793-juillet 1794), master 1, ss dir. B. Hours, 2007.

Hadj Hammar Myriam, L'organisation financière des diocèses et la fiscalité ecclésiastique locale dans le ressort de la chambre supérieure des décimes de Lyon (1693-1788), master 2, ss dir. B. Hours, 2007.

Martin Florian, La vente des biens nationaux de première origine dans le district d'Autun, master 1, ss dir. B. Hours, 2007.

Maury Serge, Discours et prophéties convulsionnaires à la fin du XVIII siècle : l'exemple du groupe fareiniste à travers les discours de la Sœur Élisée, master 2, ss dir. Y. Krumenacker, 2007. 
Moratalla Magali, Les Prost : étude d'une dynastie de notaires dans l'Ain, master 1, ss dir. B. Hours, 2007.

Noureux Arnaud, Urbanisme réglementaire et opérationnel à Lyon de 1763 à 1790, master 1, ss dir. Y. Krumenacker, 2007.

\section{8}

Genessay Jérémie, Les cloches et la Révolution dans le département de l'Ain, master 1, ss dir. P. Chopelin, 2008.

Peney Floraine, Prison et prisonniers d'Ambronay sous la Terreur, master 1, ss dir. P. Chopelin, 2008.

Simien Côme, Les massacres de septembre 1792 à Lyon, master 1, ss dir. P. Chopelin, 2008.

\section{MONTPELLIER \\ Université Paul-Valéry (Montpellier III) \\ 2007}

Lévy Anne-Laure, La caricature sous la Révolution française : le regard de Jacques-Marie Boyer Brun de Nîmes (1789-1792), master 1, ss. dir. S. Brunet, 2007.

\section{8}

Lévy Anne-Laure, Un journal local en 1789 : Le Journal de Nismes, écho public et publié des événements et débats révolutionnaires, master 2, ss. dir. S. Brunet, 2008.

\section{NANCY \\ UNIVERSITÉ NANCY 2 \\ 2004}

Furgaut Jean-Baptiste, La vie religieuse à Neufchâteau, 1690-1790, maîtrise, ss dir. Ph. Martin, 2004.

Grillon Laetitia, La légende napoléonienne en Meurthe, 1815-1870, maîtrise, ss dir. J. El Gammal, 2004.

Schwindt Frédéric, La communauté et la foi, confréries et société dans l'ouest de l'espace lorrain, XIII ${ }^{e}$-XIX $X^{e}$ siècles, thèse NR, ss dir. L. Châtellier, 2004.

\section{5}

Bihaki Grégori, Les gardes nationales de la Meurthe sous l'Empire, maîtrise, ss dir. J.-P. Rothiot, 2005.

Humbertclaude Brice, Ventron, un village vosgien pendant la Révolution, mâ̂trise, ss dir. J.-P. Rothiot, 2005.

Lang Jean-Bernard, La justice criminelle sous l'Ancien régime dans la généralité de Metz, 1744-1780, thèse NR, ss dir. Ph. Martin, 2005. 
Streiff Jean-Paul, Espaces, réseaux et sociétés urbaines de l'Ancien Régime à la Restauration. Bar-le-Duc-Commercy (1750-1820), thèse NR, ss dir. F. Roth, 2005.

Walter Jeffry, Recherches sur la vie religieuse à Épinal, 1690-1789, maîtrise, ss dir. S. Simiz, 2005.

\section{6}

Burteaux Cyrille, L'épitaphier de Nicolas Guédon, 1755, maîtrise, ss dir. Ph. Martin, 2006.

Colin Marie-Hélène, Les saints lorrains : entre religion et identité régionale (fin $X V I^{e}$-fin XIX $X^{\mathrm{e}}$ siècle), thèse NR, ss dir. Ph. Martin, 2006.

Peroz Anne, Pourvoir aux finances : la vente des biens nationaux à Épinal (de 1789 à 1827), master 2, ss dir. C. Dugas de la Boissonny, 2006.

Poirier Christelle, L'alimentation à Nancy au XVIII ${ }^{e}$ siècle, DEA, ss dir. Ph. Martin, 2006.

\section{7}

Malglaive Amélie, La Révolution française et les pauvres : un projet de bienfaisance nationale, l'exemple d'un district de la Meurthe : Blâmont, master 1, ss dir. J.-P. Rothiot, 2007.

Marso Christophe, Gorze, histoire d'un village de Moselle sous la Révolution, master 1, ss dir. J.-P. Rothiot, 2007.

Martischang François-Xavier, Les fêtes royales dans le département de la Meurthe sous la Restauration et la monarchie de Juillet, 1814-1848, master 1 et master 2, ss dir. J. El Gammal, 2006 et 2007.

Mougel Laura, Initiatives, acteurs et enjeux de l'éducation populaire à Nancy (1840-1914), master 1, ss dir. J. El Gammal, 2007.

Thiry Célia, La résistance des Espagnols lors du premier siège de Saragosse, master 1, ss dir. C. Metzger, 2007.

\section{8}

Brenneur Mathieu, Suspects et opposants politiques dans le département de la Meurthe de 1815 à 1870, master 1, ss dir. J. El Gammal, 2008.

Gerique-Valero Conception, Vivre la Révolution ou vivre sous la Révolution à Cirey-sur-Vezouze, département de la Meurthe, 29 juillet 1789-8 pluviôse an II, master 2, ss dir. J.-P. Rothiot et D. Francfort, 2008.

Herbillot Hugues, Étudier la vie d'une municipalité sous le Consulat et l'Empire, l'exemple de Neufchâteau, master 1, ss dir. J.-P. Rothiot, 2008.

Mougel Laura, L'éducation populaire et ouvrière à Nancy (1840-1914), master 2, ss dir. J. El Gammal, 2008. 


\section{PARIS \\ Université de Paris I (Panthéon-Sorbonne) \\ 2007}

Archimbaud Gilles, Bernard (de Saintes), un parcours politique (1751-1818), master 2, ss. dir. J.-C. Martin, P. Serna, 2007.

Baudry Sonia, L'espion anglais ou correspondance en Milord all'eyre and Milord all ear : une nouvelle à la main de Pidansat de Mairobert, master 1, ss dir. P. Serna, 2007.

Bernard brunel Léandre, Le mélodrame et la fantasmagorie, master 1, ss. dir. J.-C. Martin, 2007.

Bihan Benoist, La naissance et le développement des hussards en France, 16931763. Un exemple du renouveau de la cavalerie dans les armées occidentales à l'époque moderne. master 1, ss. dir. B. Gainot, 2007.

Blaisot Benjamin, Influence de l'auvre fortifiée de Vauban aux Amériques. L'exemple de la forteresse de San Juan de Ulina dans le port de Vera Cruz, Golfe du Mexique du XVI siècle au XIX siècle. master 1, ss. dir. B. Gainot, 2007.

Bodard Gwénaëlle, L'occupation française des États pontificaux (1798-1799 et 1809-1814). Le maintien de l'ordre dans le Patrimoine. De la relation entre les troupes et la population, master 2, ss. dir. B. Gainot, G. Pecout, 2007

Cancellieri Héloise, Les conditions de la formation de l'Armée Royaliste de Puisaye et ses contradictions, 1794-1795, master 1, ss. dir. J.-C. Martin, 2007

Denglos Guillaume, Iconographie et historiographie sur et autour de Condorcet, master 1, ss. dir. J.-C. Martin, 2007.

Di mauro Luca, Le prince Guiolamo Pignatelli Di Moliterno : une perspective pour l'étude du sectarisme politique dans l'Italie napoléonienne, master 2, ss. dir. P. Serna, B. Gainot, 2007.

Duhaussay Romain, Victimes et survivants des Massacres de septembre, master 1, ss. dir. J.-C. Martin, 2007.

Fernandez Julien, Du gendarme à la ville au gendarme pour la ville : la gendarmerie à Paris pendant la Révolution française (1793-1795), master 2, ss. dir. B. Gainot, V. Denis, 2007.

Foucault Hélène, L'Historien de Dupont de Nemours : la recherche du " juste milieu » ou la République conservatrice vu par un physiocrate (an IV-an V), master 1, ss. dir. P. Serna, 2007.

Gantier Vanessa, Police, espionnage et maintien de l'ordre dans la société civile des départements romains, 1810/1813, master 1, ss. dir. B. Gainot, 2007.

Hanquez Matthieu, L'affaire des naufragés de Calais, Contribution à l'histoire de l'émigration militaire, master 2, ss. dir. B. Gainot, J.-C. Martin, 2007. 
Leblois Jacques, Hippolyte Taine, historien de la Révolution française, master 2, ss. dir. J.-C. Martin, J.-L. Chappey, 2007.

Le calvez Stéphanie, La réorganisation du monde des Lettres en l'an III, master 1, ss. dir. J.-L. Chappey, 2007.

Lecam Jean-Baptiste, Éducation physique, société et politique de la veille de la révolution française à 1793, Master 1, ss. dir. J.-C. Martin, 2007.

Legrand Aurélien, La base de données : un outil pour servir à l'histoire du Tribunal révolutionnaire de Paris, master 2, ss. dir. J.-C. Martin, P. Serna, 2007.

Liechtmanger-lepitre Marie-Sophie, Le service de santé à l'Hôtel des Invalides, 1788/1800, master 2, ss. dir. B. Gainot, H. Drévillon, M. Roucaud, 2007.

Mandelbaum Stéphane, L'émergence du concept d'Outre-mer. Illustration à travers la prise et la perte de Gorée (nivôse-ventôse au XII), master 1, ss. dir. B. Gainot, 2007.

Markovic Momcilo, Répression et ordre public à Paris : 1789-1793, master 2, ss. dir. J.-C. Martin, P. Serna, 2007.

Mazeau Guillaume, Charlotte Corday et l'attentat contre Marat : événements, individus et écriture de l'histoire (1793-2007), thèse NR ss dir. J.-C. Martin, 2007.

Pauc Florence, La levée du milieu provinciale à Melun. Entre responsabilité et solidarité (1688 à 1713), master 1, ss. dir. B. Gainot, 2007.

Pezavant Lucie, Etre journaliste sous le Directoire, master 1, ss. dir. P. Serna, 2007.

Pondard Audrey, L'« univers » de la mode sous le Directoire, master 1, ss. dir. J.-C. Martin, 2007.

Quintard Domitille, Jouer pendant la Révolution française. L'exemple de la loterie dans le contexte politique, social et administratif, master 1, ss. P. Serna, 2007.

Reynier Quentin, Pierre-Jean Audoin (1764-1808). Biographie intellectuelle, master 1, ss. dir. P. Serna, 2007.

Rouille Mailys, Félicité de Choiseul Meuse un écrivain de romans érotiques au XIXe siècle. master 1, ss. dir. J.-C. Martin, 2007.

Sayette Karine, L'entrée en Révolution d'un écrivain du Chevalier Michel de Cubières, Master 1, ss. dir. J.-L. Chappey, 2007.

Vire Delphine, L'Année des Dames nationales de Nicolas-Edme Restif de la Bretonne, master 1, ss. dir. J.-C. Martin, 2007.

Wetzel Nicolas, Le renseignement militaire sur la frontière des Pyrénées durant le règne de Louis XIV, master 2, ss. dir. $\mathrm{B}$. Gainot, $\mathrm{H}$. Drevillon, M. Roucaud, 2007. 


\section{8}

Ayad-bergounioux Soulef, Antoine Boulay de la Meurthe (1761-1840) une figure de la bourgeoisie libérale et conservatrice, master 2, ss. dir. P. Serna, J.-C. Martin, 2008.

Bihan Benoist, La naissance et le développement des Hussards en France (16931763). Un exemple du renouveau de la cavalerie dans les armées occidentales, master 2, ss. dir. B. Gainot, H. Drevillon, 2008.

Bocher Héloise, Le patriote Palloy et ses réseaux, master 2, ss. dir. J.-C. Martin, P. Serna, 2008.

Danguy des Deserts Anne, Les élèves-officiers de l'École spéciale militaire de Fontainebleau, 1802-1808, master 1, ss. dir. B. Gainot, 2008.

Delaunay Léa, Critique et représentations de la société révolutionnaire, master 1, ss. dir. J.-C. Martin, 2008.

Duflo Chloé, Espace(s) politique(s) des villes de Guadeloupe de 1783 à 1794, master 1, ss. dir. B. Gainot, 2008.

Faure Marianne, Le " censeur des journaux 》 (11 fructidor an III-18 fructidor an V), master 1, ss. dir. P. Serna, 2008.

Faure Pauline, Le travail patrimonial en Révolution : acteurs et activités de la Commission temporaire des arts en 1792-1793, master 1, ss. dir. J.-L. Chappey, 2008.

Guinier Arnaud, Corps et âmes, la question disciplinaire de la paix d'Aix-laChapelle à la Révolution, master 2, ss. dir. B. Gainot, H. Drevillon, 2008.

Kechichian Jeremy, Les prémices de l'unification italienne dans les armées Napoléoniennes, master 1, ss. dir. B. Gainot, 2008.

Khodabandehlou Farzad, Souveraineté et langage politique dans le procès du roi, master 1, ss. dir. J.-C. Martin, 2008.

Lacourarie Émilie, Félicie de Fauveau, master 1, ss. dir. J.-C. Martin, 2008.

Mamere Marie, La police de Paris, 1789-1790, master 1, ss. dir. P. Serna, 2008.

Mandelbaum Stéphane, L'intégration des domaines coloniaux dans la stratégie globale des guerres du second XVIII' siècle (1750-1810) : la mise en réseau de l'information et du commandement, master 2, ss. dir. B. Gainot, L. Henninger, 2008.

Marouche Mustapha, Le rôle de l'artillerie française à la bataille d'Averstaëdt (14 octobre 1806), master 1, ss. dir. B. Gainot, 2008.

Martoire Jeanne-Laure, Police et écriture policière. Le discours policier sur la conspiration vendémiaire-ventôse an IX (hiver 1800-1801), master 1, ss. dir. B. Gainot, 2008.

Masson Catherine, Vendée et République souvenir de la Révolution et politisation des campagnes au XIX siècle, master 2, ss. dir. J.-C. Martin, J.-L. Chappey, 2008. 
Pondard Audrey, Une définition de la noblesse sous la Révolution française : la réception de la loi du 27 germinal an II, master 2, ss. dir. J.-C. Martin, J.-L. Chappey, 2008.

Reynier Quentin, La mort hérö̈que sous le Directoire, master 2, ss. dir. P. Serna, B. Gainot, 2008.

Rodriguezares Adrien, Aspects militaires de la campagne de Corse (1768-1769), master 1, ss. dir. B. Gainot, 2008.

Sarthou Adrien, Les revues militaires dans la Prusse de Frederic II (1740-1786), réalités et représentations, master 1, ss. dir. B. Gainot, 2008.

Sauphanor Axelle, Église et esclavage : les missions de Nicolas Jacquemin en Guyane, 1777-1819, master 1, ss. dir. B. Gainot, 2008.

Valour Vincent, Sublime et Révolution française. Étude du « moment robespierriste » de la Révolution, master 1, ss dir. J.-C. Martin, 2008.

\section{PARIS \\ Université Paris X-Nanterre 2007}

Baringthon Sabrina, La question coloniale dans la Chronique de Paris, master 1, ss dir. M. Belissa, 2007.

Le Roux Rémi, Les relations franco-toscanes pendant le Directoire, master 1, ss dir. M. Belissa, 2007.

\section{8}

Bégaud Stéphane, La Chine mise en Lumières, Représentations et instrumentalisation du monde chinois dans l'Encyclopédie et les publications savantes du XVIII siècle (1751-1786), master 2, ss dir. M. Belissa, 2008.

Nemraoui Samira, Les relations franco-saxonnes dans les Instructions aux Ambassadeurs 1748-1763, master 1, ss dir. M. Belissa, 2008.

Taquet Pauline, La neutralité danoise vue par la presse sous le Consulat, master 1, ss dir. M. Belissa, 2008.

\section{PAU \\ Université de PaU \\ 2008}

Espinosa-Dassonneville Gonzague, Le roi, le royaume, les affaires de France dans L'Espion chinois d'Ange Goudar (1764), master 1, ss dir. F. Bidouze et G. Lahouati, 2008, 338 p.

Malet Damien, L'Ancien régime et ses suppôts dans le Patriote français de Jacques-Pierre Brissot de Warville (1789-1793), master 1, ss dir. F. Bidouze, et A. Kouvouama, 2008, t.1, 292 p., t. 2, 105 p. 


\section{ROUEN \\ UNIVERSITÉ DE ROUEN \\ 2005}

Caru Kévin, Les almanachs et la Révolution française, master 1, ss dir. P. Dupuy et M. Biard, 2005.

Cornu Mickael, Jefferson en France : analyse du film et portée historique, master 1 , ss dir. P. Dupuy et M. Biard, 2005.

Doray Marc, L'écho de la Révolution américaine dans les débats publics en France sous la Révolution, master 1, ss dir. P. Dupuy et M. Biard, 2005.

Duclos Céline, Les comités de surveillance dans les cantons de Montivilliers, Harfleur et Le Havre (1793-1794), master 1, ss dir. M. Biard et P. Dupuy, 2005.

Fareh Mejdi, Les Égyptiens et les Français : regards croisés et relations pendant et après l'Expédition d'Égypte, thèse NR, ss dir. J.-P. Jessenne, 2005.

Roquigny Thimotée, La Société des études robespierristes et les Annales historiques de la Révolution française de 1958 à 2005, master 1, ss dir. M. Biard et P. Dupuy, 2005.

\section{6}

Dannebey Valentine, Jacobins, jacobinisme, quel écho sur l'Internet?, master 1, ss dir. M. Biard et P. Dupuy, 2006.

Deneuve Mélanie, Les répercussions du Concordat sur l'Église réformée de France : l'exemple de l'Église réformée de Rouen au début du XIX siècle, master 1, ss dir. Y. Marec et P. Dupuy, 2006.

Duclos Céline, Les comités de surveillance dans les cantons de Montivilliers, Harfleur et Le Havre (1793-1794), master 2, ss dir. M. Biard et P. Dupuy, 2006.

Legoff Jean-Baptiste, Dénoncer un représentant du peuple (septembre 1792-brumaire an IV), master 2, ss dir. M. Biard et P. Dupuy, 2006.

Roquigny Thimotée, La Société des études robespierristes et les Annales historiques de la Révolution française de 1958 à 2005, master 2, ss dir. M. Biard et P. Dupuy, 2006.

\section{7}

Abenzoar Éliane, La correspondance des membres de l'Académie des sciences, belles-lettres et arts de Rouen de 1744 à 1793, thèse NR, ss dir. Ch. Le Bozec, 2007.

Atineaulle Gaelle, La Normandie à travers les récits des voyageurs anglais lors $d u$ Grand Tour, master 1, ss dir. P. Dupuy et M. Biard, 2007.

Claim Geoffroy, La compagnie de maréchaussée de Haute-Normandie et ses héritières : les compagnies de Gendarmerie nationale de Seine-Inférieure et de l'Eure (1778-1792), master 1, ss dir. M. Biard et P. Dupuy, 2007. 
Coury Fanny, La Révolution américaine à travers les manuels scolaires $d u$ $X I X^{\mathrm{e}}$ siècle, master 1, ss dir. P. Dupuy et M. Biard, 2007.

Devillers Fanny, L'orientalisme dans les manuels scolaires $d u$ XIX $X^{\mathrm{e}}$ siècle, master 1, ss dir. P. Dupuy et M. Biard, 2007.

Goubert Gaëtan, L'administration du district de Dieppe. Étude sur les correspondances, master 1, ss dir. M. Biard et P. Dupuy, 2007.

Gricourt Mathieu, Marie-Antoinette de Sofia Coppola : entre histoire, engouement et mode médiatiques?, master 1, ss dir. M. Biard et P. Dupuy, 2007.

Hue Guillaume, La justice de paix à Quincampoix, master 1, ss dir. M. Biard et P. Dupuy, 2007.

Legoff Jean-Baptiste, Dénoncer un représentant du peuple (septembre 1792-brumaire an $(V)$, thèse de l'École des Chartes, ss dir. M. Biard, 2007.

Lemarchand Laurent, La monarchie absolue entre deux âges : épreuves, expériences et réalisations de la Régence (1715-1723), thèse NR, ss dir. J.-P. Jessenne, 2007.

Maisonnable Gaëlle, La presse féminine en France de 1789 à 1791, master 1, ss dir. M. Biard et P. Dupuy, 2007.

Méron Manon, La représentation des femmes durant la révolution française : entre violence (5-6 octobre 1789) et patriotisme (14 juillet 1790), master 1, ss dir. P. Dupuy et M. Biard, 2007.

Mouillot Fabrice, Les pamphlets révolutionnaires (1787-1795) du fonds Chardey de la Bibliothèque municipale du Havre, master 1, ss dir. M. Biard et P. Paumier, 2007.

Neusy Aurélie, Les violences populaires dans la presse et les pamphlets de la Révolution française, 1789-1792, master 1, ss dir. M. Biard et P. Dupuy, 2007.

Pencreach Annaïg, Le cheval en Normandie au XVIII ${ }^{\mathrm{e}}$ siècle, master 1, ss dir. M. Biard et P. Dupuy, 2007.

Podeur Clément, L'Angleterre dans les débats législatifs en France (1789-1793), master 1, ss dir. P. Dupuy et M. Biard, 2007.

Ponsot Armelle, Des réformes monarchiques à la Bienfaisance nationale : la mise en ouvre des politiques sociales entre Seine et Somme (1764-1795), thèse NR, ss dir. J.-P. Jessenne, 2007.

Prudhomme Marie-Claire, Entre Saint-Saens et Cailly : territoires, relations et pouvoirs dans les campagnes normandes (XVII ${ }^{e}-X V I I I^{e}$ siècles), thèse NR, ss dir. J.-P. Jessenne, 2007.

Saillard Romain, Les freins et résistances aux levées d'hommes en Seine-Inférieure (1793-1800), master 1, ss dir. M. Biard et P. Dupuy, 2007.

Silighini Réjane, La représentation des philosophes français du XVIII siècle dans les manuels scolaires $d u X I X^{\mathrm{e}}$ et $d u X X^{\mathrm{e}}$ siècle, master 1, ss dir. P. Dupuy et M. Biard, 2007. 
Vitry Héloïse, Les pièces de théâtre du fonds Chardey de la Bibliothèque municipale du Havre, master 1, ss dir. M. Biard et P. Dupuy, 2007.

\section{8}

Bous Kevin, Des " extrêmes » encore méconnus? Enragés et Hébertistes dans l'historiographie de la Révolution française, master 1, ss dir. M. Biard et P. Dupuy, 2008.

Catté Agathe, Enfer et enfers dans les pamphlets et la presse pendant la Révolution française, master 1, ss dir. M. Biard et P. Dupuy, 2008.

Chaventré Karim, La Seine-Inférieure vue de l'Assemblée nationale, master 1, ss dir. M. Biard et P. Dupuy, 2008.

Claim Geoffroy, La compagnie de maréchaussée de Haute-Normandie et ses héritières : les compagnies de gendarmerie nationale de Seine-Inférieure et de l'Eure (1778-1792), master 2, ss dir. M. Biard et P. Dupuy, 2008.

Deneuve Mélanie, Les répercussions du Concordat sur l'Église Réformée de France : l'exemple de l'Eglise réformée de Rouen au début du XIX siècle, master 1, ss dir. Y. Marec et P. Dupuy, 2008.

Dorléan Éric, Le costume créole dans les Antilles françaises au XVIII siècle, master 1, ss dir. M. Biard et P. Dupuy, 2008.

Goubert Gaëtan, L'administration du district de Dieppe. Étude sur les correspondances, master 2, ss dir. M. Biard et P. Dupuy, 2008.

Guillaume Christophe, La critique théâtrale dans la Chronique de Paris, master 1, ss dir. M. Biard et P. Dupuy, 2008.

Lefebvre Julie, Entre discours et passage à l'acte, la Terreur à Rouen, master 1, ss dir. M. Biard et P. Dupuy, 2008.

Malbire Alric, Comprendre La Révolution française. Entre histoire érudite et histoire partisane, master 1, ss dir. P. Dupuy et M. Biard, 2008.

Méron Manon, La représentation des femmes durant la Révolution française: entre violence (5-6 octobre 1789) et patriotisme (14 juillet 1790), master 2, ss dir. P. Dupuy et M. Biard, 2008.

Mouillot Fabrice, Les pamphlets révolutionnaires (1788-1795) du fonds Chardey de la Bibliothèque municipale du Havre, master 2, ss dir. M. Biard et P. Paumier, 2008.

Neusy Aurélie, Les violences populaires dans la presse et les pamphlets de la Révolution française, 1789-1792, master 2, ss dir. M. Biard et P. Dupuy, 2008.

Pencreach Annaïg, Le cheval en Normandie au XVIII ${ }^{\mathrm{e}}$ siècle, master 2, ss dir. M. Biard et P. Dupuy, 2008.

Podeur Clément, L'Angleterre dans les débats législatifs en France (1789-1793), master 2, ss dir. P. Dupuy et M. Biard, 2008. 
Roussel Anthony, Les "échos » des révoltes dans l'ouest à la Convention nationale, master 1, ss dir. M. Biard et P. Dupuy, 2008.

Silighini Réjane, La représentation des philosophes français du XVIII siècle dans les manuels scolaires $d u X I X^{\mathrm{e}}$ et $d u X X^{\mathrm{e}}$ siècle, master 2, ss dir. P. Dupuy et M. Biard, 2008.

\section{TOURS}

\section{Université François Rabelais}

Delléa Sylvain, "CEil du gouvernement » ou main de l'État? Louis Texier-Olivier, commissaire central du Directoire auprès du département d'Indre-etLoire, d'après sa correspondance avec les ministères de l'Intérieur et de la Police. Frimaire an IV-Prairial an VI (automne 1795-Printemps 1798), master 1, ss dir. N. Coquery, 2008.

\section{VALENCIENNES \\ Université de VALENCIENNES ET dU HainaUt-CAMBrÉSIS \\ 2007}

Blas Pauline, La noblesse valenciennoise dans la tourmente révolutionnaire (1770-1799), master 1, ss dir. C. Albertan, 2007.

Blas Jeanne, Entre noblesse et négoce, les Hamoir (1747-1789), master 2, ss dir. C. Albertan, 2007

Huart Nicolas, Haspres au XVIII siècle et sous la Révolution, master 1, ss dir. J. Bernet, 2007.

Lefebvre Julien, Saulzoir dans la Révolution, master 1, ss dir. C. Albertan, 2007.

Legrand Ludovic, La Terreur dans le Cambrésis, master 1, ss dir. C. Albertan, 2007.

Senez Yohan, La formation de Maximilien Robespierre, master 1, ss dir. C. Albertan, 2007.

\section{8}

Legrand Ludovic, La Terreur à Cambrai, Sûreté générale et justice révolutionnaire (10 août 1792-9 Thermidor an II), master 2, ss dir. C. Albertan, 2008. 